\title{
Designing effective questions for classroom response system teaching
}

By: Ian D. Beatty, William J. Gerace, William J. Leonard, and Robert J. Dufresne.

Beatty, I. D., Leonard, W. J., Gerace, W. J., \& Dufresne, R. J. (2006). "Designing effective questions for classroom response system teaching." American Journal of Physics 74(1) 31-39.

Made available courtesy of American Association of Physics Teachers: http://dx.doi.org/10.1119/1.2121753

****) American Association of Physics Teachers. Reprinted with permission. No further reproduction is authorized without written permission from American Association of Physics Teachers. This version of the document is not the version of record. Figures and/or pictures may be missing from this format of the document. $* * *$

Copyright (C) 2013 All rights reserved.

American Journal of Physics is copyrighted. Individual articles in American Journal of Physics are copyrighted by the society, as indicated on each article.

Individual articles may be downloaded for personal use; users are forbidden to reproduce, republish, redistribute, or resell any materials from this journal in either machine-readable form or any other form without permission of the society or payment of the appropriate royalty for reuse.

Contact rights@aip.org

\begin{abstract}
:
Classroom response systems can be powerful tools for teaching physics. Their efficacy depends strongly on the quality of the questions. Creating effective questions is difficult and differs from creating exam and homework problems. Each classroom response system question should have an explicit pedagogic purpose consisting of a content goal, a process goal, and a metacognitive goal. Questions can be designed to fulfill their purpose through four complementary mechanisms: directing students' attention, stimulating specific cognitive processes, communicating information to the instructor and students via classroom response systemtabulated answer counts, and facilitating the articulation and confrontation of ideas. We identify several tactics that are useful for designing potent questions and present four "makeovers" to show how these tactics can be used to convert traditional physics questions into more powerful questions for a classroom response system. (C) 2006 American Association of Physics Teachers.
\end{abstract}

Keywords: Physics education | kinematics | learning | room acoustics | decision analysis

Article: 


\section{Introduction}

An electronic classroom response system (CRS) such as eInstruction CPS, InterWrite (formerly EduCue) $P R S$, and $H$-ITT can be a powerful tool for physics instruction. ${ }^{1-6}$ It is merely a tool, however, not a "magic bullet." To significantly impact student learning, a CRS must be employed with skill in the service of sound and coherent pedagogy. This use is not easy.

We have helped high school teachers learn to teach physics with a CRS and studied their progress and difficulties.7-9 We have also used a CRS in our own university physics teaching, helped others learn to do so, and designed and tested CRS questions for more than ten years. 1 Our experience spans a broad array of contexts: high school classes, introductory university classes for nonscience and science majors, upper-level university classes for physics majors, and workshops for in-service physics teachers and science graduate students. We have used a CRS with classes ranging in size from fewer than 20 to over 200 students. We've taught traditional physics material as well as conceptual physics, general science, and science pedagogy.

Learning to operate the technology is the easiest part of becoming facile with CRS-based instruction. More difficult challenges include creating and adapting suitable questions, cultivating productive classroom discourse, and integrating CRS use with the rest of the course, with curricular materials, and with external constraints. ${ }^{9}$

Many who try teaching with a CRS discover that creating or finding "good" questions is more difficult than it first appears. The characteristics of effective CRS questions are very different from those of good exam questions, homework problems, and in-class worked examples. The vast archives of questions and problems that instructors accumulate over years of teaching or find in standard textbooks offer little assistance to the new CRS user. Few collections specifically designed for CRS-based teaching exist.

One relatively widely known collection of CRS questions is that contained in Eric Mazur's book Peer Instruction. ${ }^{3}$ Mazur has popularized CRS use in physics instruction, and the questions in his book are a useful starting point. However, we have found this collection to be insufficient for two reasons. One reason is that the questions have been designed to support Mazur's particular goals for CRS use and are not optimal for the more ambitious approach we use and advocate. Mazur's "peer instruction" method consists of brief lecture-style presentations on key points of physics, each followed by a short conceptual question. Students are asked to formulate their own answers and then convince their peers of the correctness of their answer. Mazur argues that this process "forces students to think though the arguments being developed, and ... provides them (as well as the teacher) with a way to assess their understanding of the concept." 3 He also suggests that knowledge "diffuses" among the students, helping to spread correct ideas.

Although this way of using a CRS is viable and valuable, we find that even more dramatic improvements in teaching and learning can occur by inverting the paradigm. Rather than following mini lectures with CRS-based quizzing, we use a CRS-powered "question cycle" as the core of in-class instruction, making question posing, pondering, answering, and discussing the vehicle of learning. Microlectures are injected only occasionally and when immediate circumstances warrant. Furthermore, we use a CRS to develop more than just conceptual 
understanding. We also target the development of cognitive skills, analysis, and problem solving ability, and productive student metacognition about physics, learning, and thinking. Our approach, called question-driven instruction, is summarized in Sec. II A and described in more detail elsewhere. ${ }^{1,8,10,11}$

The other reason that the questions in Mazur3 and similar collections are insufficient (although useful) is that instructors cannot use a question effectively if they do not appreciate its goals and design logic. They often fail to take advantage of the question's latent potential or unwittingly sabotage its effect. To use a CRS effectively, instructors need a generalized understanding of what makes CRS questions succeed or fail, how to evaluate questions, and how to invent or adapt questions to meet their personal situation, objectives, and style. Instructors should know how to create and modify, not just use, CRS questions.

Although a few isolated question-design techniques have been developed and publicized, ${ }^{12}$ we are aware of no comprehensive or systematic framework for developing and evaluating CRS questions. This paper addresses that need.

Section II summarizes our vision of the central role that CRS use can play within physics instruction and lays out a framework for thinking about and designing CRS questions. Section III describes a selection of specific tactics that can be employed when designing questions. Section IV presents four "makeovers:" examples of traditional physics questions, together with improved variations that implement some of the tactics of Sec. III. Section V summarizes the paper and offers some closing comments.

\section{THEORY: GOALS AND MECHANISMS}

A general framework for thinking about question design must address the role that the questions will play within a course, the specific goals a question can be designed to attain, and the various mechanisms by which it can attain them.

\section{A. Role: What part do questions play?}

We advocate a model of CRS-based teaching that we call "question-driven instruction." In this model, posing questions via CRS does more than augment traditional instruction: it forms the very core of the instructional dynamic. Our primary in-class goal is not to lecture or present information. Rather, we seek to help students explore, organize, integrate, and extend their knowledge. Students receive their primary exposure to new material from textbooks, multimedia, and other out-of-class resources.

In-class activity is organized around a question cycle (Fig. 1). ${ }^{8}$ We begin the cycle by presenting a question or problem to the class, generally without preamble, and allow a few minutes for students to discuss it in small groups. Typically, students within a group will argue their various opinions and intuitions, work out a solution if required, and continue discussing and elaborating until satisfied with their answer. Students then key in their responses. We view and display an instant histogram showing the class-wide distribution of responses. Without revealing which 
responses are superior, we then moderate a class-wide discussion, asking for volunteers to explain the reasoning behind each. With deft management, this process can be turned into a lively interchange of ideas and arguments between students. Based on the thinking students reveal during discussion, we can follow up with general observations, a brief microlecture, a related CRS question, or whatever else is necessary for closure; at this point, a few well-chosen comments can often precipitate significant learning. We typically repeat the entire cycle three or four times per 50-min class.

Three aspects of the cycle are worth stressing. First, questions are presented to students in a way that encourages significant cogitation, rather than just memory recall or execution of practiced skills. Second, questions are accompanied by extensive discussion: within small groups before answers are collected and by the whole class afterward. Third, the instructor continually probes for and adjusts to the students' learning needs - a practice we call "agile teaching."

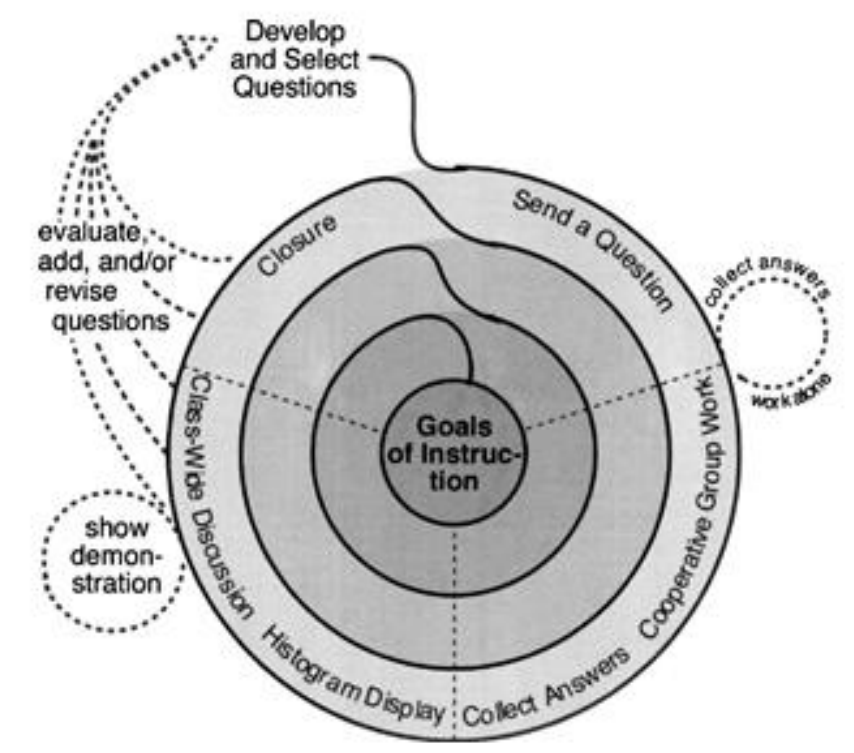

Fig. 1. The question cycle used for question-driven instruction with a clasgroom response system.

\section{B. Goals: What should the question accomplish?}

We strongly believe that every CRS question used in class should serve an explicit pedagogic objective. By "pedagogic objective," we mean more than just a particular piece of physics content. For maximum benefit, we maintain that every question should have a threefold purpose consisting of a content goal, a process goal, and a metacognitive goal.

Content goal: A question's content goal is determined by answering the question, "What piece(s) of the subject material do we want to illuminate?" This dimension of a question's purpose is the most obvious and needs little discussion other than to suggest that concepts, principles, and their inter-relationships make the best material for productive questions. Because question-driven instruction cannot explore all aspects of a subject in the classroom time allotted to a typical course, we focus question cycle iterations on the foundational ideas, core principles, crucial 
distinctions, and conceptual organization of the material at hand. With a robust understanding of these ideas, students are well prepared to learn advanced topics and special cases through reading and homework assignments.

Process goal: A question's process goal is chosen by answering the question, "What cognitive skills do we want students to exercise?" If the content goal refers to what physics material students must use to answer the question, the process goal refers to how they must use it. One might also call a process goal a "cognitive goal."

In addition to knowledge about physics, expert physicists possess a wide range of skills that make their knowledge useful in various situations. We have identified 12 habits of mind that successful physicists practice and that students should be encouraged to develop. ${ }^{8}$ For convenience, we separate them into basic and advanced sets as summarized in Table I. A question's process goal can be characterized by the habits of mind it exercises.

A crucial activity for students, spanning and integrating many habits of mind, is analysis: understanding a situation by identifying the essential concepts and their relationships and reasoning with these to draw conclusions. ${ }^{13}$ The practice of analysis develops robust conceptual understanding and connects it to successful problem-solving ability. As part of their process goal, questions should frequently require students to perform qualitative analysis in pursuit of an answer.

Metacognitive goal: A question's metacognitive goal is chosen by answering the question, "What beliefs about learning and doing physics do we wish to reinforce?" Everything that occurs within a course expresses, explicitly or implicitly, a perspective on physics, thinking, learning, teaching, and how the educational "game" should be played. Some of the many issues that this goal includes are the following: Is physics about memorizing and applying rules and equations or about reasoning and making sense of the physical world? Should students study and work in isolation or is learning a cooperative and social process? Is attention to detail important or is getting the general idea sufficient? Should conscientious and able students expect to understand material the first time it is presented, or is confusion, resolution, and multipass learning necessary? How much self-directed activity should learning physics require? The answers to these questions may be obvious to most instructors, but they are not to students; consistency of message is crucial.

The more constructive students' metacognitive perspective is, the more efficiently they can learn what we are trying to teach. By influencing their perspective, we can significantly enhance learning in our courses and help to prepare students for future learning throughout and beyond school. And recent thinking on the transfer of knowledge suggests that "preparation for future learning" is the most durable learning outcome our instruction is likely to achieve. ${ }^{14,15}$ 


\begin{tabular}{ll}
$\begin{array}{l}\text { Table I. "Habits of mind" that expert physicists possegs and students should } \\
\text { develop. }\end{array}$ \\
\hline \hline Basic & Advanced \\
\hline Seek alternative representations & Generate multiple solutions \\
Compare and contrast & Categorize and classify \\
Explain, describe, and depict & Discuss, summarize, and model \\
Predict and observe & Strategize, justify, and plan \\
Extend the context & Reflect and evaluate \\
Monitor and refine communication & Think about thinking and learning \\
\hline
\end{tabular}

\section{Mechanisms: How can a question accomplish its goals?}

A question-driven instruction question can fulfil its pedagogic purpose by four different general mechanisms or "channels:" through focusing students' attention by posing the question, through stimulating cognitive processes as students ponder the question, through feedback provided to students and instructor by collective viewing of the response histogram, and through articulation and confrontation of ideas during discussion.

Questions can have a very powerful effect on students merely by being posed and pondered. The first two mechanisms can be thought of as "What are they thinking about?" and "How are they thinking about it?" A question can direct students' attention to specific facts, features, ideas, conflicts, or relationships, bringing issues to conscious awareness. Sometimes merely looking at an idea, or looking at it from the right angle, is enough to spark understanding. Other times, it is a necessary but insufficient first step. Also, a question can stimulate students to exercise specific cognitive processes: habits of mind and the general practice of analysis. No question can force students to engage in any particular cognitive process; mental engagement is always voluntary. However, the design of a question can necessitate certain processes to reach a successful answer and can make the need for certain processes relatively obvious.

The third and most obvious mechanism by which a CRS question can serve pedagogic ends is by communicating information about student responses. By seeing the histogram of answers entered, the instructor learns about students' understanding and students learn about their classmates' thinking. If this information is not merely noted, but actually used by the instructor or students to make subsequent teaching and learning decisions, then response system use constitutes formative assessment: assessment to enhance, rather than evaluate, learning. Formative assessment is perhaps the most effective instructional "innovation" ever studied. ${ }^{16-20}$

Interstudent and student-instructor discussion is the fourth mechanism by which questions can fulfil their design objectives. The discussion that accompanies use of a question - within small groups before answering and class-wide after the histogram is displayed - is crucial to effective question- driven instruction. One reason is that the act of articulating beliefs, perceptions, assumptions, expectations, understanding, and reasoning is inherently valuable to students. Thinking is often ill-formed, nebulous, and inconsistent. When a student must cast such thinking into language, especially the precise language of physics, such deficiencies become evident and must be redressed. 
Another reason is that discussion involves a confrontation of different perceptions, different analyses, and different conclusions. Exposing students to their classmates' thinking challenges their own and promotes learning. Arguing and reconciling differences promotes yet more. Telling students what to think is notoriously ineffective; eliciting their thinking, confronting it with alternatives, and seeking resolution works better.

Yet another reason is that whole-class discussion can reveal to the instructor far more about students' understanding and difficulties than any single histogram, no matter how informative the question's distracters. We use these discussions to actively inform ourselves of the nature and causes of our students' errors. This discussion is also a kind of formative assessment.

\section{TACTICS: IMPLEMENTING THE THEORY}

Section II presented a general framework for thinking about the design of questions for questiondriven instruction using a classroom response system. In this section, we present some specific tactics (listed in Table II) that can be used to implement the framework. These are merely a helpful, representative set; others certainly exist. They have been grouped according to which of the four mechanisms they employ. Our convention is to present tactic names in italics and habits of mind "in quotes." (Some habits of mind are also tactic names; the formatting indicates which is meant in a particular context.) For many of the tactics, we have indicated which of the makeovers in Sec. IV employ them.

\section{A. Tactics for directing attention and raising awareness}

Removing nonessentials from a question is a general, obvious, and often neglected tactic for focusing students' attention where we want it. By this tactic, we mean removing anything nonessential to the instructor's pedagogic purpose for the question (not necessarily to the students' efforts to answer it). For example, we avoid having potentially distracting features in the question's situation or potentially distracting steps in the thinking students must do to reach an answer. A question with a qualitative point, for example, is only weakened by requiring quantitative calculations that may distract students and divert their cognitive resources. Makeover C in Sec. IV demonstrates this tactic. 
Table II. Question design tactics.

Tactics for directing attention and raising awareness:

Remove nonessentials

Compare and contrast

Extend the context

Reuse familiar question situations

Oops-go-back

Tactics for stimulating cognitive processes:

Interpret representations

Compare and contrast

Extend the context

Identify a set or subset

Rank variants

Reveal a better way

Strategize only

Include extraneous information

Omit necessary information

Tactics for formative use of response data:

Answer choices reveal likely difficulties

Use "none of the above"

Tactics for promoting articulation discussion:

Qualitative questions

Analysis and reasoning questions

Multiple defensible answers

Require unstated assumptions

Trap unjustified assumptions

Deliberate ambiguity

lrollmg for misconceptions

a) A cannon attached to a train fires in the direction the train is moving. At what angle above the horizontal should it fire so that the projectile lands as far as possible from where it is fired?

1) much greater than $45^{\circ}$ 4) slightly under $45^{\circ}$

2) slightly over $45^{\circ}$

3) exactly $45^{\circ}$

5) much less than $45^{\circ}$

6) Not enough information

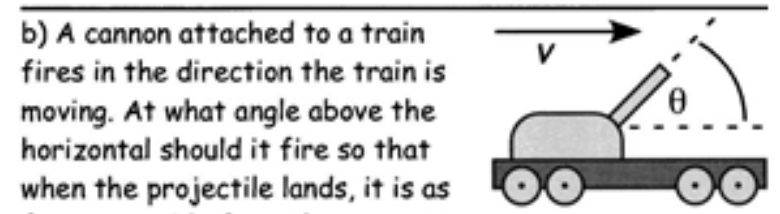
far as possible from the cannon?

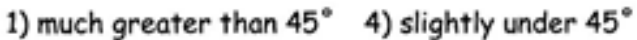

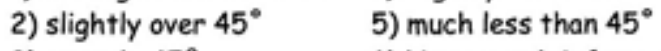

3) exactly $45^{\circ} \quad$ 6) Not enough information

Fig. 2. A question pair exemplifying the oops-go-back design tactic. 
Compare and contrast is another tactic for directing attention and awareness. By having students compare two things, their attention will naturally be drawn to the differences between them. One way to implement this tactic is to pose a question that has students comparing multiple situations - physical arrangements, processes, conditions, etc.- to categorize or order them. Another is to describe a situation and ask about the effect of changing some aspect of it. A third is to present a sequence of two or more CRS questions, in which the situations are identical but the query varies or in which the same query is made about slightly different situations. Makeover A demonstrates a question comparing two situations.

Extending the context of a known idea or skill is a habit of mind and also a valuable question design tactic. By asking a familiar question about an unfamiliar situation, students' attention is drawn to the ways in which the new situation differs from known ones and to the relevance of these differences, and broadens students' comprehension of the relevant ideas. Students expand their understanding of concepts beyond the limited scope that they initially encounter by seeing them in varied contexts. For example, when students begin to grasp the procedure for finding the normal force of an object on a flat horizontal surface, tilt or curve the surface. When they assimilate that, add additional forces on the object or give it a nonzero acceleration. Makeover A incorporates this tactic by asking a linear dynamics question about a situation generally used for rotational dynamics.

Reusing familiar question scenarios also has its place. Reading, digesting, and interpreting a question statement requires nontrivial cognitive resources from students - resources that could be spent on understanding the points we want to make with the question. The tactic of removing nonessentials helps reduce this "cognitive load" effect, as does building new questions from situations and systems students have already come to understand. Use of the extending the context and reusing familiar question scenarios tactics should be balanced: In general, new ideas should be introduced in familiar question contexts, while somewhat familiar ideas should be explored and developed in novel contexts.

Oops-go-back is an awareness-raising tactic involving a sequence of two related questions. The first is a trap: A question designed to draw students into making a common error or overlooking a significant consideration. The instructor allows students to respond and then moves to the second question without much discussion. The second causes students to realize their mistake on the first question. When students are "burned" this way by a mistake and discover it on their own, they are far more likely to learn from it than if they are merely warned about it in advance or informed that they have committed it. A simple example that is suitable early on during coverage of kinematics would be to ask students for the velocity of some object moving in the negative direction or in two dimensions, with positive scalar answer choices including the object's speed, and also "None of the above." Many students who are insufficiently attuned to the distinction between speed and velocity will erroneously select the speed. Then, a second question asks about the object's speed, causing many students to consider how this question differs from the previous and realize their error.

This technique can be subtle, so we will present a second, less trivial example. If we ask the question in Fig. 2(a), many students will erroneously answer " 45 degrees." Without discussion, we then present the question in Fig. 2(b). Many will realize that the answer to this one is " 45 
degrees," be bothered by this answer, reconsider how the first question differs, and realize that they had neglected the cannon's velocity relative to the ground. While answering the first question, they had unwittingly answered the second.

\section{B. Tactics for stimulating cognitive processes}

Specific question design tactics for stimulating cognitive processes are as varied as the spectrum of thinking skills they target. The fundamental rule is to ask questions that cannot be answered without exercising the desired habits of mind and to avoid excess baggage that might distract students from the need to exercise them. Certain types of problems are helpful in this regard.

Many students are inordinately attached to algebraic representations of physics concepts, relationships, and situations and do not fully appreciate the utility of "alternative" representations such as graphs, free body and vector diagrams, and even verbal descriptions. Questions that require students to interpret representations are useful for decreasing this attachment and for developing the habit of mind, "seek alternative representations." The tactic is implemented by providing necessary information or answer options in an alternative representation. For example, we might describe an object's motion with a graph and then ask a question about its behavior that requires students to recognize and interpret the information latent in aspects of the graph, such as a slope or the area under a curve. Alternatively, we might ask them to verbally describe the meaning of a mathematical equation, or to choose which of a set of vectors best describes some quantity. Makeover D does not require students to interpret representations, but it does rely on the tactic for the "surprise" solution revealed during discussion (see reveal a better way, below).

The compare and contrast and extend the context tactics for focusing students' attention are also useful for developing the habits of mind of those names. These are powerful question types with multiple benefits.

Some habits of mind are easy to target. "Categorize and classify" is promoted by presenting students with a set of situations, objects, or processes and asking them to identify a set or subset meeting some criterion or to rank variants according to some quality.

Constrain the solution is a tactic for exercising the habit of mind "generate multiple solutions." This tactic can be a "positive constraint" directing students to solve a problem via some particular approach (for example, "use the work- energy theorem") or a "negative constraint" directing students not to use some particular approach (for example, "do it without solving for the acceleration"). Merely giving such a directive gets students to consider their activity from a strategic perspective.

Reveal a better way is another, less direct tactic for strengthening "generate multiple solutions." First, we present a question that students are likely to solve by a valid but difficult, tedious, errorprone, opaque, or otherwise nonoptimal path. Then, during discussion, we can suggest a dramatically more elegant or simple solution. Makeover D is a classic example of this tactic. 
Strategize only is a tactic for strengthening the habit of mind "strategize, justify, and plan." It is implemented by presenting a problem and asking students to identify the principle(s) and approach that would be most useful for reaching a solution, without actually solving the problem. This tactic teaches students to think explicitly about problem solving and the set of strategies available to them. (The "justify" portion of that habit of mind is naturally developed during discussion of students' reasoning.) Makeover $\mathrm{C}$ demonstrates this tactic.

Include extraneous information and omit necessary information are other tactics useful for developing "strategize, justify, and plan." They push students to consider explicitly what information is necessary to complete a strategy, rather than assuming every question provides exactly what is required and nothing more. (Note that include extraneous information is not inconsistent with remove nonessentials, because deliberately extraneous information can be essential to the pedagogic purpose of the question. It is extraneous to students, but not to the instructor.)

Similar tactics can be imagined for other habits of mind. Once one has decided to target a specific cognitive facility and has escaped from the trap of always using standard calculate-ananswer questions, creating suitable question types is generally straightforward.

Cognitive processes are targeted not just by the intrinsic construction of the question, but also by classroom interaction surrounding its use. Reveal a better way relies on carefully engineered classroom interaction. "Monitor and refine communication" is exercised any time communication within the classroom is explicitly discussed, perhaps after students have misinterpreted a question or a statement by the instructor. Similarly, "think about thinking and learning" (metacognition) is stimulated whenever the instructor asks students to explicitly consider their own thinking processes and learning behaviors.

\section{Tactics for formative use of response data}

As we have described, the third general mechanism by which questions can fulfill their design objectives is by providing information to the instructor and students through the histogram of students' responses. To provide maximally useful information to the instructor, questions should be designed so that answer choices reveal likely student difficulties including common errors, misunderstandings, and alternative assumptions and interpretations. In this way, we can glance at the histogram and quickly detect whether a particular one of these is prevalent in our class and decide whether to address it. In general, a response histogram is most useful to students and instructor when the spectrum of answers chosen is broad rather than narrowly peaked around one choice. (One exception is the first question of an oops-go-back pair, for which having a majority of students fall into the "trap" can be desirable.)

When interpreting students' responses, we must remember that any given answer can almost always be reached by more than one path or argument. Thus, having students explain their answers is vital. For this reason among others, we usually open the whole-class discussion for a question by proceeding systematically down the answer list, asking for (or cajoling) volunteers to present a rationale for each. We maintain a "poker face" throughout. After a student has argued for a particular answer, we ask if anyone has a different reason for the same answer. 
We frequently include "none of the above" (or "not enough information") as an answer choice, so as to learn about responses we might not have anticipated. We make this the "correct" or best answer often enough that students learn to take it seriously (often for omit necessary information) and don't assume that they have made a mistake if they don't agree with one of the other options offered.

\section{Tactics for promoting articulation, conflict, and productive discussion}

As mentioned, the fourth mechanism of question efficacy-discussion-has students learn by articulating their thinking, confronting each other's' thinking, and resolving differences. It also provides the instructor with valuable information about students' understanding confusions, progress, and predilections. Not all questions lead to equally productive discussion. Questions that are most useful for this tend to be quite different from standard exam-type questions.

Qualitative questions are usually superior to quantitative ones for promoting articulation and argument. Quantitative questions lure students into thinking in terms of numbers, variables, and equations, which are difficult to communicate and discuss; qualitative questions promote discussion in terms of concepts, ideas, and general relationships. The final question versions in all four makeovers of Sec. IV are qualitative.

Analysis and reasoning questions that require significant decision making by students similarly lead to better discussion and more valuable articulation than those requiring calculation or memory recall. (They also promote the development of analytic skills.) Makeovers B and C introduce elements of analysis, and Makeover D is a good analysis question (although it may initially seem like a straightforward algebra problem to students).

Questions with multiple defensible answers are useful for sowing dissension and generating productive discussion. Perhaps more than one answer is viable depending on how one chooses to interpret the question or on what assumptions one consciously or unconsciously makes. Makeover B exemplifies this.

Similarly, we can design questions that require unstated assumptions, trap unjustified assumptions, or contain deliberate ambiguity. In addition to promoting disagreement and therefore profitable discussion, these have the benefit of sensitizing students to the multiple interpretations possible in many situations, to the importance of assumptions in physics reasoning, and to the criteria physicists use when evaluating assumptions. Makeover D introduces an unstated assumption (leading to multiple defensible answers).

Trolling for misconceptions is another useful tactic: engineering questions that deliberately catch students in likely misconceptions or undesirable "alternative conceptions." Such questions further the content goal of helping students become aware of and escape the particular misconception, improving their physics knowledge. They further the metacognitive goal of putting students on the alert for misconceptions in general. They also tend to promote argument, sometimes impassioned. Makeovers A and B both target specific misconceptions. 
How one conducts class discussion can be more important to the quality of the discussion than what questions are used. Emphasizing cogency of reasoning over correctness of results is crucial, as is stressing that the only "bad" answer is one that does not reflect a student's actual thinking. Other, more specific tactics for moderating discussion exist, but are outside the scope of this paper.

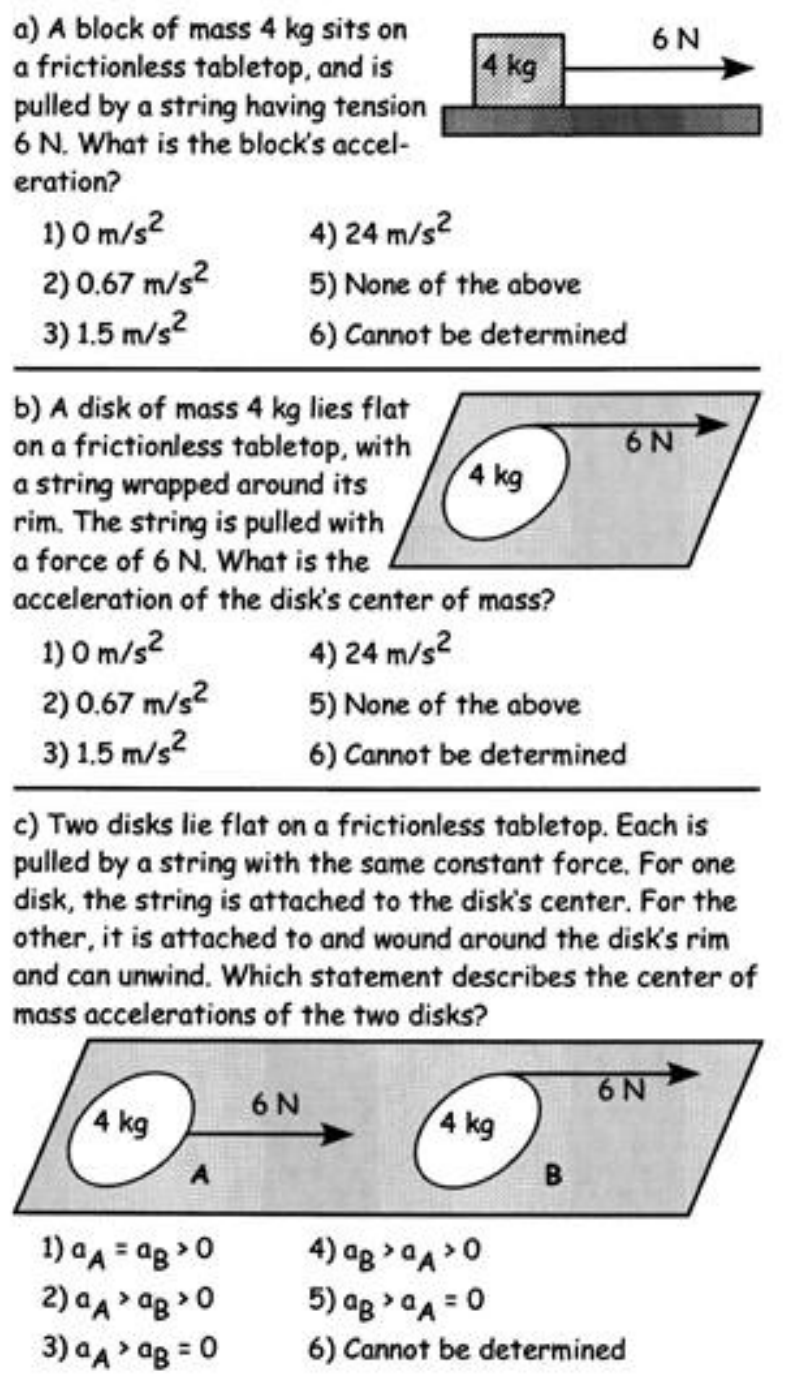

Fig. 3. Three variants of a question on Newton's second law in one dimension.

\section{EXAMPLES}

Abstract advice without concrete examples can be difficult to implement. In this section we present four "makeovers:" case studies in which a traditional question is improved by incorporating some of the question-driven instruction question design tactics. Each is accompanied by a discussion of which tactics have been implemented. 


\section{A. Newton's second law}

Figure 3(a) shows a straightforward question on Newton's second law in one dimension. The variant in Fig. 3(b) requires the same content knowledge, but incorporates the tactic of trolling for a misconception to promote disagreement and argumentation. A common misconception among novice physics students is that ' $\mathrm{T}=I$ a. somehow supersedes or modifies $F=m a$. By displaying the surface features of a rotational dynamics problem, this question will lure many students into the trap of thinking that because the disk rotates, some of the force is "used up" and the resulting linear acceleration will be less than Newton's second law alone would predict. Although requiring only Newton's second law in one linear dimension, the question would be appropriate for use after rotational dynamics has been introduced.

Note that the question wording does not explicitly ask for the linear acceleration of the disk. Most likely, some students will assume that the question is asking for angular acceleration and choose the "cannot be determined" answer. This answer choice allows the instructor to stress that "acceleration," unqualified, means "linear acceleration." If a large enough fraction of the class answers this way, we recommend clarifying the point and then starting over without further discussion and allowing students to reanswer so that the question's primary intent can be realized. (To make the variant more effective at trapping this error, the disk's radius can be given and some answer choices in appropriate units for angular acceleration can be provided.)

Figure 3(c) shows a variant incorporating yet more question design tactics. Compare and contrast is used to focus students' attention on the effect of rotational motion on linear acceleration and to practice the "compare and contrast" habit of mind. This variant is a qualitative question that trolls more effectively for the same misconception and interpretation error as the previous variant. It is a powerful tool for pushing students to articulate an intuitive misconception and to realize, wrestle with, and resolve the contradictions to which it leads. (With deft handling, an instructor can use it to compare and contrast the ideas of force and acceleration with work and energy. Although the two disks experience the same force and have the same acceleration, one gains more kinetic energy than the other in a given time interval.) The variant would have been effective and simpler if the three answer choices were $a A<a B, a A$ $=a B$, and $a A>a B$. Both are implementations of rank variants. However, the set of distracters provided in variant (c) helps the instructor distinguish between students who think the disk will accelerate more slowly when rotating and those who think it will spin without translating at all, making use of answer choices reveal likely difficulties.

\section{B. Identifying forces}

Figure 4(a) shows a question that targets students' ability to identify the forces on a body. The question's content goal is to have students appreciate that apart from gravity (and other action at a distance forces not encountered during introductory mechanics), all forces acting on a body are caused by interactions with other bodies in direct contact with it. This is a qualitative question that trolls for the misconception that contact forces can be "transmitted" through an intermediate body to act between two separated bodies. 
As written, the question is not bad, but it could be better. Consider the variant in Fig. 4(b). It is still qualitative and trolls for the same misconception. However, it is open ended and has students enumerate the forces (a modification of identify a set or subset) to uncover other, perhaps unanticipated, misconceptions and errors. For example, we might discover that some students count the "net force" alongside other forces.

More importantly, it employs the multiple defensible answers tactic: Choices 4 through 8 are all justifiable, depending on whether one treats the interaction between the plane and block as a single contact force or as two (friction and normal), whether one neglects buoyancy and drag due to air, whether one includes silly but real forces like the gravitational effect of the moon, etc. (From a microscopic perspective, one can argue for an uncountable and fluctuating number of forces due to molecular collisions.) This question could also be considered an implementation of the require unstated assumptions or deliberate ambiguity tactics. Knowing the answer that a student has chosen conveys little information about their degree of understanding or about specific confusions that they may have. Instead, the question serves to get students thinking about which forces are present without prompting them with specific forces. Then, during the whole-class discussion, the instructor can ignore the answers chosen and instead discuss various possible forces in turn, arguing whether or not each merits counting.

This variant also addresses additional content goals. During the discussion, the instructor can model a general procedure for identifying the forces on a body and illuminate the various choices and conventions involved in identifying a "force" (for example, the convention of treating one contact force between two surfaces as two distinct, orthogonal forces: the "normal" and "friction" forces). In addition, the question makes an excellent platform for discussing the role of assumptions and approximations in physics and helping students learn when to include or neglect various forces.

Perhaps the most powerful aspect of this variant is its effectiveness at achieving the metacognitive goal of communicating to students that they should be concerned with reasoning, learning, and the cogency of their answers, and not with the correctness or incorrectness of any particular answer choice. Most students are deeply attached to the notion that every question has a "right" or "best" answer. We have found that the only way to make students abandon this attachment and really focus on reasoning is to use questions like Fig. 4(b), for which it is obvious that several answers are defensible and can be correct or incorrect depending on the argument behind them. 
a) A mass $m$ slides down a frictionless circular track of radius $R$. What is its angular velocity relative to the center of curvature just as it reaches the bottom?
1) $\sqrt{(2 g R)}$
2) $-\sqrt{ }(2 g R)$
5) $\int(2 g / R)$
3) $\sqrt{ }(2 g R)$
6) 0
7) None of the above
4) $\sqrt{ }(g / 2 R)$
8) Not enough information

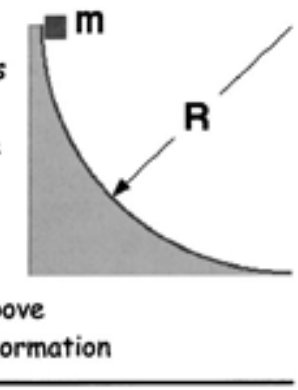
b) A mass $m$ slides down a frictionless circular track of radius $R$. Which of the following would let you most efficiently find its angular velocity relative to the center of curvature when it reaches the bottom?

1) Kinematics only

2) $F=m a$ or Newton's laws

3) Work-energy theorem

4) Impulse-momentum theorem

5) Angular impulse-angular momentum theorem

6) More than one of the above

7) None of the above

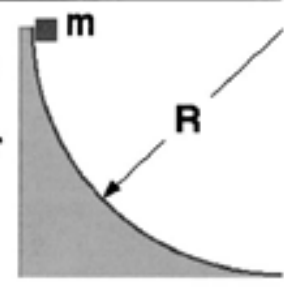

Fig. 5. Two variants of a question on strategic choices in problem solving.

a) A marble moving at $40 \mathrm{~cm} / \mathrm{s}$ rolls onto a horizontal piece of felt and stops after 1.25 seconds. Assuming constant acceleration, how far does it travel on the felt?
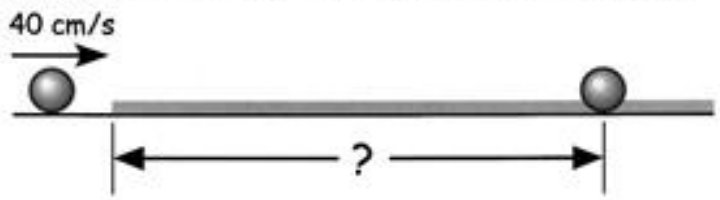

1) $25 \mathrm{~cm}$

4) $75 \mathrm{~cm}$

2) $42.3 \mathrm{~cm}$

5) None of the above

3) $50 \mathrm{~cm}$

6) Not enough information

b) A marble rolls onto a horizontal piece of felt that is $30 \mathrm{~cm}$ in length. After $20 \mathrm{~cm}$, the marble's speed is half its initial value. Does the marble reach the far edge of the felt?

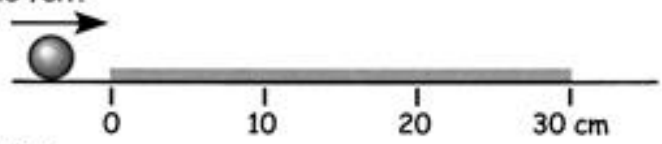

1) Yes.

2) No.

3) Not enough information.

Fig. 6. Two variants of a kinematics question. 


\section{Energy and angular motion}

Figure 5(a) shows a question that helps students integrate their knowledge by requiring a mix of energy and angular motion ideas. To answer the question correctly, students must recognize the need for conservation of energy, apply that principle, and relate linear to angular motion. The variant in Fig. 5(b) improves on the original by incorporating several question design tactics. Because the question's primary content goal is to develop students' ability to recognize the need for two different strategic steps using physics from two distinct topic areas (energy conservation and relating linear to angular motion), this variant is a qualitative question that removes nonessentials to focus students' attention more effectively; students rarely pay sufficient attention to high-level, strategic aspects of problem solving when embroiled in equation manipulation. It uses the strategize only tactic. The question's phrase "most efficiently" can be considered a deliberate ambiguity: Is efficiency defined in terms of number of lines of calculation required, number of principles involved, intricacy of thought entailed, or something else? Does efficiency depend on the skills of the learner? (If the question initiates a class-wide discussion of "efficiency" in problem solving, so much the better.)

\section{Kinematics}

Figure 6(a) is a relatively straightforward kinematics question. It is nontrivial in that students aren't given the acceleration and must determine it first before calculating the distance traveled. Alternatively, students can determine the average velocity and multiply it by the time. The question requires some strategic decision making.

The variant in Fig. 6(b) is similar in content. If approached algebraically, it is also similar in difficulty. However, this variant is a qualitative question, making it more amenable to analysis and reasoning and more suitable for discussion. By omitting the statement "Assuming a constant acceleration," we have employed the require unstated assumptions tactic (and multiple defensible answers as a result) and opened up the possibility of discussing whether and how the constancy of the acceleration matters.

More interestingly, this variant permits the instructor to reveal a better way of answering the question. Assume constant acceleration and sketch a graph of velocity versus time. By identifying the area under the line (the time integral) with the distance traveled, one can use simple geometry to see that when the marble has slowed to half its original velocity, it has traveled three-quarters of the distance it will cover before coming to rest. If it reaches half speed after $20 \mathrm{~cm} / \mathrm{s}$, it must stop at 4/3 that distance, which is before the end of the felt: This alternate solution involves interpreting representations, and the variant is a better analysis and reasoning question than variant (a).

\section{SUMMARY AND REMARKS}


In the question-driven instruction approach, the use of a classroom response system to pose, collect answers for, and discuss questions forms the core of in-class instruction. The success of the approach depends in part on the quality of the questions used. Effective questions should be designed with an explicit, threefold pedagogic objective consisting of a content goal, a process (cognitive) goal, and a metacognitive goal. The content goal is the topic material to be addressed. It should generally be conceptual and foundational in nature and should frequently integrate ideas from various portions of the curriculum. The process goal is the set of cognitive skills to be developed and can be thought of in terms of 12 "habits of mind" and the general practice of qualitative analysis. The metacognitive goal is the perspective about learning or physics to be reinforced.

A question can fulfil its threefold pedagogic objective through four complementary mechanisms. The posing of the question can focus students' attention on particular issues. Students' pondering of the question can stimulate particular cognitive skills. Displaying the answer histogram can convey information about student knowledge and thinking to class- mates and to the instructor. Discussion, both small group and whole class, can impact students and inform the teacher as students struggle to articulate and defend their thinking and confront others' perceptions, interpretations, assumptions, and reasoning.

Questions can be deliberately engineered for maximal learning. We have identified some tactics such as remove nonessentials, compare and contrast, interpret representations, and strategize only that can be used in the design of powerful questions. Often a question in the standard style can be improved through minor modifications that take ad-vantage of one or more of these tactics.

Even with an explicit framework such as the one presented here, designing effective questions is challenging and time consuming, and like any other nontrivial skill requires practice. A repository of well-designed questions can be very helpful, and we have made many of our questions available through a Web site ${ }^{21}$ and an annotated commercial product. ${ }^{22}$ However, to teach most effectively with another person's questions, it is necessary to understand the goals and design logic of each one. ${ }^{9}$ The framework presented here helps to analyze existing questions as well.

We reiterate that well-designed questions are merely a tool, one component of the questiondriven instruction approach. Pedagogy - how the instructor uses questions to interact with students in the classroom - is more important. ${ }^{8,10,11}$ Nevertheless, a lack of effective questions can be a serious and frustrating barrier to teachers seeking to learn and practice question-driven instruction. We believe the framework and question-design tactics presented here can help overcome this barrier.

Although a classroom response system is a tremendously useful tool for implementing questiondriven instruction, it is not essential to the philosophy of question-driven instruction. Many of the ideas presented here can be productively employed without technology, especially in small, highly inter- active classes. 
Question-driven instruction is based on formative assessment. By its very nature, formative assessment tends to be self-correcting: The feedback it provides to the practitioner about student learning can, if studied attentively, reveal implementation weaknesses and improve practice over time. Therefore, our most important piece of advice regarding question-driven instruction is to pay critical attention to what happens when you do it. Your students are your best teachers.

\section{ACKNOWLEDGMENTS}

The ideas and perspectives presented in this paper have been developed during research projects supported by National Science Foundation Grant Nos. DUE-9453881, ESI-9730438, and ROLE0106771.

1. R. J. Dufresne, W. J. Gerace, W. J. Leonard, J. P. Mestre, and L. Wenk, “Classtalk: A classroom communication system for active learning," J. Comput. High. Educ. 7, 3-47 (1996).

2. R. Hake, "Interactive-engagement versus traditional methods: A six- thousand-student survey of mechanics test data for introductory physics courses," Am. J. Phys. 66, 64-74 (1998).

3. E. Mazur, Peer Instruction: A User's Manual (Prentice-Hall, Upper Saddle River, NJ, 1997).

4. W. R. Penuel, J. Roschelle, V. Crawford, N. Shechtman, and L. Abrahamson, "CATAALYST workshop report: Advancing research on the transformative potential of interactive pedagogies and classroom net- works," Workshop Report P14566, SRI International (2004).

5. J. Roschelle, W. R. Penuel, and L. Abrahamson, "The networked class- room," Educ. Leadership 61, 50-54 (2004).

6. D. Zollman and N. S. Rebello, "The evolving classroom response system at KSU: Classtalk, PRS, PDAs," contributed talk EG08 at the 130th Na- tional Meeting of the American Association of Physics Teachers (Albuquerque, NM, 2005).

7. R. J. Dufresne and W. J. Gerace, “Assessing-to-learn: Formative assessment in physics instruction," Phys. Teach. 42 (7), 428-433 (2004).

8. R. J. Dufresne, W. J. Gerace, J. P. Mestre, and W. J. Leonard, “ASK-IT/ A2L: Assessing student knowledge with instructional technology," Technical Report UMPERG-2000-09 (University of Massachusetts Physics Education Research Group, 2000).

9. Feldman and B. Capobianco, "Real-time formative assessment: A study of teachers' use of an electronic response system to facilitate serious discussion about physics concepts," Annual Meeting of the American Educational Research Association (Chicago, IL, 2003). 
D. Beatty, "Transforming student learning with classroom communication systems," Research Bulletin ERB0403, Educause Center for Applied Research (2004).

11. Beatty, W. J. Leonard, W. J. Gerace, and R. J. Dufresne, "Question driven instruction: Teaching science (well) with an audience response system," in Audience Response Systems in Higher Education: Applications and Cases, edited by D. A. Banks (Idea Group, Hershey, PA, in press).

12. P. Li, N. W. Reay, and L. Bao, "Effects of in-class polling on student performance in learning physics," contributed poster CO26 at the 129th National Meeting of the American Association of Physics Teachers, Sacramento, CA (2004).

13. W. J. Leonard, W. J. Gerace, and R. J. Dufresne, "Analysis-based problem solving: Making analysis and reasoning the focus of physics instruction," Technical Report UMPERG-2001-12 (University of Massachusetts Physics Education Research Group, 2001).

14. J. D. Bransford and D. Schwartz, "Rethinking transfer: A simple proposal with multiple implications," in Review of Research in Education, edited by A. Iran-Nejad and P. D. Pearson (American Educational Research Association, Washington, DC, 1999), Vol. 24, pp. 61-100.

15. J. D. Bransford, A. L. Brown, and R. R. Cocking, How People Learn: Brain, Mind, Experience, and School (National Academy Press, Washing- ton, DC, 1999).

16. B. Bell and B. Cowie, "The characteristics of formative assessment in science education," Sci. Educ. 85, 536-553 (2001).

17. P. Black and D. William, "Assessment and classroom learning," Assess- ment in Education 5, 7-74 (1988).

18. P. Black and D. William, "Inside the black box: Raising standards through classroom assessment," Phi Delta Kappan 80, 139-147 (1988).

19. C. Boston, "The concept of formative assessment," Technical Report ED470206, ERIC Clearinghouse on Assessment and Evaluation (2002).

20. E. H. Hobson, "Formative assessment: An annotated bibliography," The Clearing House MMPI Publ. 71 (2), 123-125 (1997). 
21. I. D. Beatty, "Assessing-to-Learn Physics: Project Website," (http://A2L.physics.umass.edu/)

22. I. D. Beatty, W. J. Leonard, and W. J. Gerace, Assessing-to-Learn in the Classroom (Thomson Learning, 2005) (http://physics.brookscole.com/a2lc) 